\title{
On the Dirichlet problem for a class of nonlinear degenerate elliptic equations
}

\author{
Albo Carlos Cavalheiro* \\ Department of Mathematics, State University of Londrina, Londrina - PR, Brazil
}

(Received: 12 June 2020. Received in revised form: 10 August 2020. Accepted: 10 August 2020. Published online: 12 August 2020.)

(c) 2020 the author. This is an open access article under the CC BY (International 4.0) license (www.creativecommons.org/licenses/by/4.0/).

\begin{abstract}
In this paper, we prove the existence and uniqueness of solutions for a class of nonlinear degenerate elliptic equations $L u(x)=f_{0}(x)-\sum_{j=1}^{n} D_{j} f_{j}(x)$ in the setting of the weighted Sobolev spaces, where $D_{j}=\partial / \partial x_{j}$ and $L$ is a second order degenerate elliptic operator in divergence form in a bounded open subset of $\mathbb{R}^{n}$.
\end{abstract}

Keywords: nonlinear degenerate elliptic equations; weighted Sobolev spaces.

2020 Mathematics Subject Classification: 35J70, 35J60, 35J30.

\section{Introduction}

We are interested in the existence of (weak) solutions in the weighted Sobolev space $W_{0}^{1, p}\left(\Omega, \omega_{1}, \omega_{2}\right)($ see Definition 2.2$)$ for the Dirichlet problem

$$
(P)\left\{\begin{array}{l}
L u(x)=f_{0}(x)-\sum_{j=1}^{n} D_{j} f_{j}(x) \text { in } \Omega, \\
u(x)=0 \text { on } \partial \Omega
\end{array}\right.
$$

where

$$
L u(x)=-\operatorname{div}\left[\mathcal{A}(x, u, \nabla u) \omega_{1}+\mathcal{B}(x, u, \nabla u) \nu_{1}\right]+\mathcal{H}(x, u, \nabla u) \nu_{2}+|u|^{p-2} u \omega_{2},
$$

$D_{j}=\partial / \partial x_{j}, \Omega$ is a bounded open set in $\mathbb{R}^{n} ; \omega_{1}, \omega_{2}, \nu_{1}$ and $\nu_{2}$ are four weight functions (which represent the degeneration or singularity in Equation (1)), $1<q, s<p<\infty$ and the functions $\mathcal{A}_{j}: \Omega \times \mathbb{R} \times \mathbb{R}^{n} \rightarrow \mathbb{R}, \mathcal{B}_{j}: \Omega \times \mathbb{R} \times \mathbb{R}^{n} \rightarrow \mathbb{R}(j=1, \ldots, n)$ and $\mathcal{H}: \Omega \times \mathbb{R} \times \mathbb{R}^{n} \rightarrow \mathbb{R}$ satisfy the following conditions:

(H1). $x \mapsto \mathcal{A}_{j}(x, \eta, \xi)$ is measurable on $\Omega$ for all $(\eta, \xi) \in \mathbb{R} \times \mathbb{R}^{n} ;(\eta, \xi) \mapsto \mathcal{A}_{j}(x, \eta, \xi)$ is continuous on $\mathbb{R} \times \mathbb{R}^{n}$ for almost all $x \in \Omega$.

(H2). There exists a constant $\theta_{1}>0$ such that $\left\langle\mathcal{A}(x, \eta, \xi)-\mathcal{A}\left(x, \eta^{\prime}, \xi^{\prime}\right),\left(\xi-\xi^{\prime}\right)\right\rangle \geq \theta_{1}\left|\xi-\xi^{\prime}\right|^{p}$, whenever $\xi, \xi^{\prime} \in \mathbb{R}^{n}, \xi \neq \xi^{\prime}$, and $\mathcal{A}(x, \eta, \xi)=\left(\mathcal{A}_{1}(x, \eta, \xi), \ldots, \mathcal{A}_{n}(x, \eta, \xi)\right)$, where $\langle.,$.$\rangle denotes the Euclidian scalar product in \mathbb{R}^{n}$.

(H3). $\langle\mathcal{A}(x, \eta, \xi), \xi\rangle \geq \lambda_{1}|\xi|^{p}$, where $\lambda_{1}$ is a positive constant.

(H4). $|\mathcal{A}(x, \eta, \xi)| \leq K_{1}(x)+h_{1}(x)|\eta|^{p / p^{\prime}}+h_{2}(x)|\xi|^{p / p^{\prime}}$, where $K_{1}, h_{1}$ and $h_{2}$ are nonnegative functions, with $h_{1}, h_{2} \in L^{\infty}(\Omega)$ and $K_{1} \in L^{p^{\prime}}\left(\Omega, \omega_{1}\right)$ provided that $1 / p+1 / p^{\prime}=1$.

(H5). $x \mapsto \mathcal{B}_{j}(x, \eta, \xi)$ is measurable on $\Omega$ for all $(\eta, \xi) \in \mathbb{R} \times \mathbb{R}^{n} ;(\eta, \xi) \mapsto \mathcal{B}_{j}(x, \eta, \xi)$ is continuous on $\mathbb{R} \times \mathbb{R}^{n}$ for almost all $x \in \Omega$.

(H6). $\left\langle\mathcal{B}(x, \eta, \xi)-\mathcal{B}\left(x, \eta^{\prime}, \xi^{\prime}\right),\left(\xi-\xi^{\prime}\right)\right\rangle>0$, whenever $\xi, \xi^{\prime} \in \mathbb{R}^{n}, \xi \neq \xi^{\prime}$, where $\mathcal{B}(x, \eta, \xi)=\left(\mathcal{B}_{1}(x, \eta, \xi), \ldots, \mathcal{B}_{n}(x, \eta, \xi)\right)$.

(H7). $\langle\mathcal{B}(x, \eta, \xi), \xi\rangle \geq \lambda_{2}|\xi|^{q}+\Lambda_{2}|\eta|^{q}$, where $\lambda_{2}>0$ and $\Lambda_{2} \geq 0$ are constants.

(H8). $|\mathcal{B}(x, \eta, \xi)| \leq K_{2}(x)+g_{1}(x)|\eta|^{q / q^{\prime}}+g_{2}(x)|\xi|^{q / q^{\prime}}$, where $K_{2}, g_{1}$ and $g_{2}$ are nonnegative functions, with $g_{1}, g_{2} \in L^{\infty}(\Omega)$, and $K_{2} \in L^{q^{\prime}}\left(\Omega, \nu_{1}\right)$ provided that $1 / q+1 / q^{\prime}=1$.

(H9). $x \mapsto \mathcal{H}(x, \eta, \xi)$ is measurable on $\Omega$ for all $(\eta, \xi) \in \mathbb{R} \times \mathbb{R}^{n} ;(\eta, \xi) \mapsto \mathcal{H}(x, \eta, \xi)$ is continuous on $\mathbb{R} \times \mathbb{R}^{n}$ for almost all $x \in \Omega$.

(H10). $\left[\mathcal{H}(x, \eta, \xi)-\mathcal{H}\left(x, \eta^{\prime}, \xi^{\prime}\right)\right]\left(\eta-\eta^{\prime}\right)>0$, whenever $\eta, \eta^{\prime} \in \mathbb{R}, \eta \neq \eta^{\prime}$.

(H11). $\mathcal{H}(x, \eta, \xi) \eta \geq \lambda_{3}|\xi|^{s}+\Lambda_{3}|\eta|^{s}$, where $\lambda_{3}$ and $\Lambda_{3}$ are nonnegative constants.

\footnotetext{
*Email address: accava@gmail.com
} 
(H12). $|\mathcal{H}(x, \eta, \xi)| \leq K_{3}(x)+h_{2}(x)|\eta|^{s / s^{\prime}}+h_{3}(x)|\xi|^{s / s^{\prime}}$, where $K_{3}, h_{2}$ and $h_{3}$ are nonnegative functions, with $K_{3} \in L^{s^{\prime}}\left(\Omega, \nu_{2}\right)$, $1 / s+1 / s^{\prime}=1$ and $h_{2}, h_{3} \in L^{\infty}(\Omega)$.

By the symbol $\mathcal{W}(\Omega)$ we denote the set of all those positive and finite functions $\omega=\omega(x), x \in \Omega$, that are measurable almost everywhere in $\Omega$. Elements of $\mathcal{W}(\Omega)$ will be called weight functions. Every weight $\omega$ gives rise to a measure on the measurable subsets of $\mathbb{R}^{n}$ through integration. This measure will be denoted by $\mu$. Thus, $\mu(E)=\int_{E} \omega(x) d x$, for any measurable set $E \subset \mathbb{R}^{n}$.

In general, the Sobolev spaces $W^{k, p}(\Omega)$ without weights occur as spaces of solutions for elliptic and parabolic partial differential equations. For degenerate partial differential equations, i.e., equations with various types of singularities in the coefficients, it is natural to look for solutions in weighted Sobolev spaces (see [2-4,7]). In various applications, we may meet boundary value problems for the elliptic equations whose ellipticity is disturbed in the sense that some degeneration or singularity appears. There are several very concrete problems from practice which lead to such differential equations, e.g. from glaceology, non-Newtonian fluid mechanics, flows through porous media, differential geometry, celestial mechanics, climatology, petroleum extraction and reaction-diffusion problems (see some examples of applications of degenerate elliptic equations in $[1,6])$.

A well-known class of weights is the class of $A_{p}$-weights (or Muckenhoupt class) that was introduced by Muckenhoupt [15]. This class of weights has found many useful applications in harmonic analysis, see [18]. Another reason for studying $A_{p}$-weights is the fact that powers of distance to submanifolds of $\mathbb{R}^{n}$ often belong to $A_{p}$, see [13]. There are, in fact, many interesting examples of weights (see [12] for p-admissible weights).

The following theorem will be proved in Section 3.

Theorem 1.1. Let $1<q, s<p<\infty$ and assume that the conditions (H1)-(H12) hold. If

(i) $\omega_{1}, \omega_{2} \in A_{p}, \nu_{1}, \nu_{2} \in \mathcal{W}(\Omega), \frac{\nu_{1}}{\omega_{1}} \in L^{r_{1}}\left(\Omega, \omega_{1}\right)$ and $\frac{\nu_{2}}{\omega_{1}} \in L^{r_{2}}\left(\Omega, \omega_{1}\right)$, where $r_{1}=p /(p-q)$ and $r_{2}=p /(p-s)$;

(ii) $f_{0} / \nu_{1} \in L^{q^{\prime}}\left(\Omega, \nu_{1}\right)$ and $f_{j} / \omega_{1} \in L^{p^{\prime}}\left(\Omega, \omega_{1}\right)$ for $j=1, \ldots, n$;

then the problem (P) has a unique solution $u \in W_{0}^{1, p}\left(\Omega, \omega_{1}, \omega_{2}\right)$. Moreover, we have

$$
\|u\|_{W_{0}^{1, p}\left(\Omega, \omega_{1}, \omega_{2}\right)} \leq C\left(C_{p, q}\left\|f_{0} / \nu_{1}\right\|_{L^{q^{\prime}}\left(\Omega, \nu_{1}\right)}+\sum_{j=1}^{n}\left\|f_{j} / \omega_{1}\right\|_{L^{p^{\prime}}\left(\Omega, \omega_{1}\right)}\right)^{1 /(p-1)},
$$

where $C=\left[\left(C_{\Omega}^{p}+1\right) / \lambda_{1}\right]^{1 /(p-1)}, C_{\Omega}$ is the constant in Theroem 2.2 and $C_{p, q}$ is the constant in Remark 2.2(i).

\section{Definitions and basic results}

Let $\omega$ be a locally integrable nonnegative function in $\mathbb{R}^{n}$ and assume that $0<\omega<\infty$ almost everywhere. We say that $\omega$ belongs to the Muckenhoupt class $A_{p}, 1<p<\infty$, or that $\omega$ is an $A_{p}$-weight, if there is a constant $C=C_{p, \omega}$ such that

$$
\left(\frac{1}{|B|} \int_{B} \omega(x) d x\right)\left(\frac{1}{|B|} \int_{B} \omega^{1 /(1-p)}(x) d x\right)^{p-1} \leq C,
$$

for all balls $B \subset \mathbb{R}^{n}$, where $|$.$| denotes the n$-dimensional Lebesgue measure in $\mathbb{R}^{n}$. If $1<q \leq p$, then $A_{q} \subset A_{p}$ (see $[10,12,18]$ for more information about $A_{p}$-weights). The weight $\omega$ satisfies the doubling condition if there exists a positive constant $C$ such that $\mu(B(x ; 2 r)) \leq C \mu(B(x ; r))$, for every ball $B=B(x ; r) \subset \mathbb{R}^{n}$, where $\mu(B)=\int_{B} \omega(x) d x$. If $\omega \in A_{p}$, then $\mu$ is doubling (see Corollary 15.7 in [12]).

Let us consider an example of $A_{p}$-weight. For $x \in \mathbb{R}^{n}$, the function $\omega(x)=|x|^{\alpha}$ is in $A_{p}$ if and only if $-n<\alpha<n(p-1)$ (see Corollary 4.4 of Chapter IX in [18]).

If $\omega \in A_{p}$, then $\left(\frac{|E|}{|B|}\right)^{p} \leq C \frac{\mu(E)}{\mu(B)}$, whenever $B$ is a ball in $\mathbb{R}^{n}$ and $E$ is a measurable subset of $B$ (see 15.5 strong doubling property in [12]). Therefore, if $\mu(E)=0$ then $|E|=0$. The measure $\mu$ and the Lebesgue measure $|\cdot|$ are mutually absolutely continuous, i.e., they have the same zero sets $(\mu(E)=0$ if and only if $|E|=0)$; so there is no need to specify the measure when using the ubiquitous expression almost everywhere and almost every, both abbreviated "a.e.".

Definition 2.1. Let $\omega$ be a weight, and let $\Omega \subset \mathbb{R}^{n}$ be open. For $1<p<\infty$, we define $L^{p}(\Omega, \omega)$ as the set of measurable functions $f$ on $\Omega$ satisfying $\|f\|_{L^{p}(\Omega, \omega)}=\left(\int_{\Omega}|f|^{p} \omega d x\right)^{1 / p}<\infty$.

If $\omega \in A_{p}, 1<p<\infty$, then $\omega^{-1 /(p-1)}$ is locally integrable and we have $L^{p}(\Omega, \omega) \subset L_{\text {loc }}^{1}(\Omega)$ for every open set $\Omega$ (see Remark 1.2.4 in [19]). Thus, it makes sense to talk about weak derivatives of functions in $L^{p}(\Omega, \omega)$. 
Definition 2.2. Let $\Omega \subset \mathbb{R}^{n}$ be a bounded open set and let $\omega_{1}$ and $\omega_{2}$ be $A_{p}$-weights $(1<p<\infty)$. We define the weighted Sobolev space $W^{1, p}\left(\Omega, \omega_{1}, \omega_{2}\right)$ as the set of functions $u \in L^{p}\left(\Omega, \omega_{2}\right)$ with weak derivatives $D_{j} u \in L^{p}\left(\Omega, \omega_{1}\right)$. The norm of $u$ in $W^{1, p}\left(\Omega, \omega_{1}, \omega_{2}\right)$ is defined by

$$
\|u\|_{W^{1, p}\left(\Omega, \omega_{1}, \omega_{2}\right)}=\left(\int_{\Omega}|u|^{p} \omega_{2} d x+\int_{\Omega}|\nabla u|^{p} \omega_{1} d x\right)^{1 / p} .
$$

The space $W_{0}^{1, p}\left(\Omega, \omega_{1}, \omega_{2}\right)$ is the closure of $C_{0}^{\infty}(\Omega)$ with respect to the norm (2). Equipped with this norm, $W_{0}^{1, p}\left(\Omega, \omega_{1}, \omega_{2}\right)$ is a reflexive Banach space (see $[14,16]$ for more information about the spaces $W^{1, p}\left(\Omega, \omega_{1}, \omega_{2}\right)$ ). The dual of the space $W_{0}^{1, p}\left(\Omega, \omega_{1}, \omega_{2}\right)$ is the space defined as

$$
\left[W_{0}^{1, p}\left(\Omega, \omega_{1}, \omega_{2}\right)\right]^{*}=\left\{T=f_{0}-\operatorname{div}(F), F=\left(f_{1}, \ldots, f_{n}\right): \frac{f_{0}}{\omega_{2}} \in L^{p^{\prime}}\left(\Omega, \omega_{2}\right), \frac{f_{j}}{\omega_{1}} \in L^{p^{\prime}}\left(\Omega, \omega_{1}\right), j=1, \ldots, n\right\} .
$$

If $T \in\left[W_{0}^{p}\left(\Omega, \omega_{1}, \omega_{2}\right)\right]^{*}$ and $\varphi \in W_{0}^{1, p}\left(\Omega, \omega_{1}, \omega_{2}\right)$, we take

$$
(T \mid \varphi)=\int_{\Omega} f_{0} \varphi d x+\sum_{j=1}^{n} f_{j} D_{j} \varphi d x, \quad\|T\|_{*}=\left\|f_{0} / \omega_{2}\right\|_{L^{p^{\prime}}\left(\Omega, \omega_{2}\right)}+\sum_{j=1}^{n}\left\|f_{j} / \omega_{1}\right\|_{L^{p^{\prime}}\left(\Omega, \omega_{1}\right)}, \quad|(T \mid \varphi)| \leq\|T\|_{*}\|\varphi\|_{W_{0}^{1, p}\left(\Omega, \omega_{1}, \omega_{2}\right)} .
$$

If $\omega=\omega_{1}=\omega_{2}$, we denote $W_{0}^{1, p}(\Omega, \omega)=W_{0}^{1, p}(\Omega, \omega, \omega)$. To prove the main result of this paper, we use the following results.

Theorem 2.1. Let $\omega \in A_{p}, 1<p<\infty$, and let $\Omega$ be a bounded open set in $\mathbb{R}^{n}$. If $u_{m} \rightarrow u$ in $L^{p}(\Omega, \omega)$ then there exist a subsequence $\left\{u_{m_{k}}\right\}$ and a function $\Phi \in L^{p}(\Omega, \omega)$ such that

(i) $u_{m_{k}}(x) \rightarrow u(x), m_{k} \rightarrow \infty$ a.e. on $\Omega$;

(ii) $\left|u_{m_{k}}(x)\right| \leq \Phi(x)$ a.e. on $\Omega$.

Proof. The proof of this theorem follows from the lines of Theorem 2.8.1 in [9].

Theorem 2.2. (The weighted Sobolev inequality) Let $\Omega$ be an open bounded set in $\mathbb{R}^{n}$ and $\omega \in A_{p}(1<p<\infty)$. There exist positive constants $C_{\Omega}$ and $\delta$ such that for every $u \in W_{0}^{1, p}(\Omega, \omega)$ and for each $k$ satisfying $1 \leq k \leq n /(n-1)+\delta$, it holds

$$
\|u\|_{L^{k p}(\Omega, \omega)} \leq C_{\Omega}\||\nabla u|\|_{L^{p}(\Omega, \omega)},
$$

where $C_{\Omega}$ depends only on $n, p$, the $A_{p}$-constant $C(p, \omega)$ of $\omega$ and on the diameter of $\Omega$.

Proof. It suffices to prove (3) for every function $u \in C_{0}^{\infty}(\Omega)$ (see Theorem 1.3 in [8]). In order to extend the estimates (3) to an arbitrary $u \in W_{0}^{1, p}(\Omega, \omega)$, let $\left\{u_{m}\right\}$ be a sequence of $C_{0}^{\infty}(\Omega)$ functions tending to $u$ in $W_{0}^{1, p}(\Omega, \omega)$. Applying the estimates (3) to the differences $u_{m_{1}}-u_{m_{2}}$, we see that $\left\{u_{m}\right\}$ will be a Cauchy sequence in $L^{k p}(\Omega, \omega)$. Consequently, the limit function $u$ lies in the desired spaces and satisfies (3).

Remark 2.1. If $u \in W_{0}^{1, p}\left(\Omega, \omega_{1}, \omega_{2}\right)$ then by Theorem 2.2 (with $k=1$ ), it holds that

$$
\|u\|_{L^{p}\left(\Omega, \omega_{1}\right)} \leq C_{\Omega}\||\nabla u|\|_{L^{p}\left(\Omega, \omega_{1}\right)} \leq C_{\Omega}\|u\|_{W_{0}^{1, p}\left(\Omega, \omega_{1}, \omega_{2}\right)} .
$$

Hence, $W_{0}^{1, p}\left(\Omega, \omega_{1}, \omega_{2}\right) \subset W_{0}^{1, p}\left(\Omega, \omega_{1}\right)$.

Proposition 2.1. Let $1<p<\infty$.

(a) There exists a constant $C_{p}$ such that $\left.|| \xi\right|^{p-2} \xi-|\eta|^{p-2} \eta\left|\leq C_{p}\right| \xi-\eta \mid(|\xi|+|\eta|)^{p-2} \forall \xi, \eta \in \mathbb{R}^{n}$.

(b) There exist two positive constants $\beta_{p}, \gamma_{p}$ such that for every $x, y \in \mathbb{R}^{n}$, it holds that

$$
\left.\beta_{p}(|x|+|y|)^{p-2}|x-y|^{2} \leq\left\langle|x|^{p-2} x-|y|^{p-2} y\right),(x-y)\right\rangle \leq \gamma_{p}(|x|+|y|)^{p-2}|x-y|^{2} .
$$

Proof. See Proposition 17.2 and Proposition 17.3 in [5].

Definition 2.3. We say that an element $u \in W_{0}^{1, p}\left(\Omega, \omega_{1}, \omega_{2}\right)$ is a (weak) solution of problem (P) if

$$
\int_{\Omega}\langle\mathcal{A}(x, u, \nabla u), \nabla \varphi\rangle \omega_{1} d x+\int_{\Omega}\langle\mathcal{B}(x, u, \nabla u), \nabla \varphi\rangle \nu_{1} d x+\int_{\Omega} \mathcal{H}(x, u, \nabla u) \varphi \nu_{2} d x+\int_{\Omega}|u|^{p-2} u \varphi \omega_{2} d x=\int_{\Omega} f_{0} \varphi d x+\sum_{j=1}^{n} \int_{\Omega} f_{j} D_{j} \varphi d x,
$$

for all $\varphi \in W_{0}^{1, p}\left(\Omega, \omega_{1}, \omega_{2}\right)$. 
Remark 2.2. (i) If $\frac{\nu_{1}}{\omega_{1}} \in L^{r_{1}}\left(\Omega, \omega_{1}\right)$ (where $\left.r_{1}=p /(p-q), 1<q<p<\infty\right)$ then $\|u\|_{L^{q}\left(\Omega, \nu_{1}\right)} \leq C_{p, q}\|u\|_{L^{p}\left(\Omega, \omega_{1}\right)}$, where $C_{p, q}=$ $\left\|\nu_{1} / \omega_{1}\right\|_{L^{r_{1}\left(\Omega, \omega_{1}\right)}}^{1 / q}$. In fact, by Hölder's inequality we obtain

$$
\begin{aligned}
\|u\|_{L^{q}\left(\Omega, \nu_{1}\right)}^{q}=\int_{\Omega}|u|^{q} \nu_{1} d x=\int_{\Omega}|u|^{q} \frac{\nu_{1}}{\omega_{1}} \omega_{1} d x & \leq\left(\int_{\Omega}|u|^{q p / q} \omega_{1} d x\right)^{q / p}\left(\int_{\Omega}\left(\nu_{1} / \omega_{1}\right)^{p /(p-q)} \omega_{1} d x\right)^{(p-q) / p} \\
& =\|u\|_{L^{p}\left(\Omega, \omega_{1}\right)}^{q}\left\|\nu_{1} / \omega_{1}\right\|_{L^{r_{1}}\left(\Omega, \omega_{1}\right)} .
\end{aligned}
$$

Hence, $\|u\|_{L^{q}\left(\Omega, \nu_{1}\right)} \leq C_{p, q}\|u\|_{L^{p}\left(\Omega, \omega_{1}\right)}$.

(ii) Analogously, if $\frac{\nu_{2}}{\omega_{1}} \in L^{r_{2}}\left(\Omega, \omega_{1}\right)$ (where $\left.r_{2}=p /(p-s), 1<s<p<\infty\right)$ then $\|u\|_{L^{s}\left(\Omega, \nu_{2}\right)} \leq C_{p, s}\|u\|_{L^{p}\left(\Omega, \omega_{1}\right)}$, where $C_{p, s}=$ $\left\|\nu_{2} / \omega_{1}\right\|_{L^{r_{2}\left(\Omega, \omega_{1}\right)}}^{1 / s}$

\section{Proof of Theorem 1.1}

The basic idea is to reduce the problem $(\mathrm{P})$ to an operator equation $A u=T$ and apply the theorem given below.

Theorem 3.1. Let $A: X \rightarrow X^{*}$ be a monotone, coercive and hemicontinuous operator on the real, separable, reflexive Banach space $X$. Then the following assertions hold:

(a) For each $T \in X^{*}$ the equation $A u=T$ has a solution $u \in X$;

(b) If the operator $A$ is strictly monotone, then equation $A u=T$ is uniquely solvable in $X$.

Proof. See Theorem 26.A in [21].

To prove Theorem 1.1, we define $\mathbf{B}: W_{0}^{1, p}\left(\Omega, \omega_{1}, \omega_{2}\right) \times W_{0}^{1, p}\left(\Omega, \omega_{1}, \omega_{2}\right) \rightarrow \mathbb{R}$ and $\mathbf{T}: W_{0}^{1, p}\left(\Omega, \omega_{1}, \omega_{2}\right) \rightarrow \mathbb{R}$ by

$$
\mathbf{B}(u, \varphi)=\mathbf{B}_{1}(u, \varphi)+\mathbf{B}_{\mathbf{2}}(u, \varphi)+\mathbf{B}_{3}(u, \varphi)+\mathbf{B}_{4}(u, \varphi) \quad \text { and } \quad \mathbf{T}(\varphi)=\int_{\Omega} f_{0} \varphi d x+\sum_{j=1}^{n} \int_{\Omega} f_{j} D_{j} \varphi d x
$$

where $\mathbf{B}_{\mathbf{i}}: W_{0}^{1, p}\left(\Omega, \omega_{1}, \omega_{2}\right) \times W_{0}^{1, p}\left(\Omega, \omega_{1}, \omega_{2}\right) \rightarrow \mathbb{R}$, for $i=1,2,3,4$, are defined as

$$
\begin{aligned}
& \mathbf{B}_{1}(u, \varphi)=\int_{\Omega}\langle\mathcal{A}(x, \nabla u), \nabla \varphi\rangle \omega_{1} d x, \quad \mathbf{B}_{2}(u, \varphi)=\int_{\Omega}\langle\mathcal{B}(x, u, \nabla u), \nabla \varphi\rangle \nu_{1} d x, \\
& \mathbf{B}_{3}(u, \varphi)=\int_{\Omega} \mathcal{H}(x, u, \nabla u) \varphi \nu_{2} d x \text { and } \mathbf{B}_{4}(u, \varphi)=\int_{\Omega}|u|^{p-2} u \varphi \omega_{2} d x .
\end{aligned}
$$

Then $u \in W_{0}^{1, p}\left(\Omega, \omega_{1}, \omega_{2}\right)$ is a (weak) solution to problem $(\mathrm{P})$ if $\mathbf{B}(u, \varphi)=\mathbf{B}_{\mathbf{1}}(u, \varphi)+\mathbf{B}_{\mathbf{2}}(u, \varphi)+\mathbf{B}_{3}(u, \varphi)+\mathbf{B}_{4}(u, \varphi)=\mathbf{T}(\varphi)$, for all $\varphi \in W_{0}^{1, p}\left(\Omega, \omega_{1}, \omega_{2}\right)$.

Step 1. For $j=1, \ldots, n$ we define the operator $\left.F_{j}: W_{0}^{1, p}\left(\Omega, \omega_{1}, \omega_{2}\right)\right) \rightarrow L^{p^{\prime}}\left(\Omega, \omega_{1}\right)$ as $\left(F_{j} u\right)(x)=\mathcal{A}_{j}(x, u(x), \nabla u(x))$. We now show that the operator $F_{j}$ is bounded and continuous.

(i) Using (H4), Theorem 2.2 (since $\omega_{1} \in A_{p}$ ) and Remark 2.1, we obtain

$$
\begin{aligned}
\left\|F_{j} u\right\|_{L^{p^{\prime}\left(\Omega, \omega_{1}\right)}}^{p^{\prime}} & =\int_{\Omega}\left|F_{j} u(x)\right|^{p^{\prime}} \omega_{1} d x=\int_{\Omega}\left|\mathcal{A}_{j}(x, u, \nabla u)\right|^{p^{\prime}} \omega_{1} d x \leq \int_{\Omega}\left(K_{1}+h_{1}|u|^{p / p^{\prime}}+h_{2}|\nabla u|^{p / p^{\prime}}\right)^{p^{\prime}} \omega_{1} d x \\
& \leq C_{p} \int_{\Omega}\left(K_{1}^{p^{\prime}}+h_{1}^{p^{\prime}}|u|^{p}+h_{2}^{p^{\prime}}|\nabla u|^{p}\right) \omega_{1} d x=C_{p}\left[\int_{\Omega} K_{1}^{p^{\prime}} \omega_{1} d x+\int_{\Omega} h_{1}^{p^{\prime}}|u|^{p} \omega_{1} d x+\int_{\Omega} h_{2}^{p^{\prime}}|\nabla u|^{p} \omega_{1} d x\right] \\
& \leq C_{p}\left[\int_{\Omega} K_{1}^{p^{\prime}} \omega_{1} d x+\left\|h_{1}\right\|_{L^{\infty}(\Omega)}^{p^{\prime}} \int_{\Omega}|u|^{p} \omega_{1} d x+\left\|h_{2}\right\|_{L^{\infty}(\Omega)}^{p^{\prime}} \int_{\Omega}|\nabla u|^{p} \omega_{1} d x\right] \\
& \leq C_{p}\left[\left\|K_{1}\right\|_{L^{p^{\prime}\left(\Omega, \omega_{1}\right)}}^{p^{\prime}}+\left(C_{\Omega}^{p}\left\|h_{1}\right\|_{L^{\infty}(\Omega)}^{p^{\prime}}+\left\|h_{2}\right\|_{L^{\infty}(\Omega)}^{p^{\prime}}\right) \int_{\Omega}|\nabla u|^{p} \omega_{1} d x\right] \\
& \leq C_{p}\left[\left\|K_{1}\right\|_{L^{p^{\prime}\left(\Omega, \omega_{1}\right)}}^{p^{\prime}}+\left(C_{\Omega}^{p}\left\|h_{1}\right\|_{L^{\infty}(\Omega)}^{p^{\prime}}+\left\|h_{2}\right\|_{L^{\infty}(\Omega)}^{p^{\prime}(\Omega)}\right)\|u\|_{W_{0}^{1, p}\left(\Omega, \omega_{1}, \omega_{2}\right)}^{p}\right] .
\end{aligned}
$$

where the constant $C_{p}$ depends only on $p$. Therefore, in (4) we obtain

$$
\left\|F_{j} u\right\|_{L^{p^{\prime}\left(\Omega, \omega_{1}\right)}} \leq C_{p}^{1 / p^{\prime}}\left(\left\|K_{1}\right\|_{L^{p^{\prime}\left(\Omega, \omega_{1}\right)}}+\left(C_{\Omega}^{p-1}\left\|h_{1}\right\|_{L^{\infty}(\Omega)}+\left\|h_{2}\right\|_{L^{\infty}(\Omega)}\right)\|u\|_{W_{0}^{1, p}\left(\Omega, \omega_{1}, \omega_{2}\right)}^{p-1}\right) .
$$

(ii) Let $u_{m} \rightarrow u$ in $W_{0}^{1, p}\left(\Omega, \omega_{1}, \omega_{2}\right)$ as $m \rightarrow \infty$. We need to show that $F_{j} u_{m} \rightarrow F_{j} u$ in $L^{p^{\prime}}\left(\Omega, \omega_{1}\right)$. We will apply the Lebesgue Dominated Convergence Theorem. If $u_{m} \rightarrow u$ in $W_{0}^{1, p}\left(\Omega, \omega_{1}, \omega_{2}\right)$, then $\left|\nabla u_{m}\right| \rightarrow|\nabla u|$ in $L^{p}\left(\Omega, \omega_{1}\right)$. Using Theorem 2.1, there 
exist a subsequence $\left\{u_{m_{k}}\right\}$ and a function $\Phi_{1} \in L^{p}\left(\Omega, \omega_{1}\right)$ such that $u_{m_{k}}(x) \rightarrow u(x)$ a.e. in $\Omega, D_{j} u_{m_{k}}(x) \rightarrow D_{j} u(x)$ a.e. in $\Omega$, and $\left|\nabla u_{m_{k}}(x)\right| \leq \Phi_{1}(x)$ a.e. in $\Omega$. Next, applying (H4) and Theorem 2.2 we obtain

$$
\begin{aligned}
\left\|F_{j} u_{m_{k}}-F_{j} u\right\|_{L^{p^{\prime}\left(\Omega, \omega_{1}\right)}}^{p^{\prime}}= & \int_{\Omega}\left|F_{j} u_{m_{k}}(x)-F_{j} u(x)\right|^{p^{\prime}} \omega_{1} d x=\int_{\Omega}\left|\mathcal{A}_{j}\left(x, u_{m_{k}}, \nabla u_{m_{k}}\right)-\mathcal{A}_{j}(x, u, \nabla u)\right|^{p^{\prime}} \omega_{1} d x \\
\leq & C_{p} \int_{\Omega}\left(\left|\mathcal{A}_{j}\left(x, u_{m_{k}}, \nabla u_{m_{k}}\right)\right|^{p^{\prime}}+\left|\mathcal{A}_{j}(x, u, \nabla u)\right|^{p^{\prime}}\right) \omega_{1} d x \\
\leq & C_{p}\left[\int_{\Omega}\left(K_{1}+h_{1}\left|u_{m_{k}}\right|^{p / p^{\prime}}+h_{2}\left|\nabla u_{m_{k}}\right|^{p / p^{\prime}}\right)^{p^{\prime}} \omega_{1} d x\right. \\
& \left.+\int_{\Omega}\left(K_{1}+h_{1}|u|^{p / p^{\prime}}+h_{2}|\nabla u|^{p / p^{\prime}}\right)^{p^{\prime}} \omega_{1} d x\right] \\
\leq & C_{p}\left[\int_{\Omega} K_{1}^{p^{\prime}} \omega_{1} d x+\left\|h_{1}\right\|_{L^{\infty}(\Omega)}^{p^{\prime}} \int_{\Omega}\left|u_{m_{k}}\right|^{p} \omega_{1} d x+\left\|h_{2}\right\|_{L^{\infty}(\Omega)}^{p^{\prime}(\Omega)}\left|\nabla u_{m_{k}}\right|^{p} \omega_{1} d x\right. \\
& \left.+\left\|h_{1}\right\|_{L^{\infty}(\Omega)}^{p^{\prime}} \int_{\Omega}|u|^{p} \omega_{1} d x+\left\|h_{2}\right\|_{L^{\infty}(\Omega)}^{p^{\prime}} \int_{\Omega}|\nabla u|^{p} \omega_{1} d x\right] \\
\leq & 2 C_{p}\left[\left\|K_{1}\right\|_{L^{p^{\prime}}\left(\Omega, \omega_{1}\right)}^{p^{\prime}}+\left\|h_{1}\right\|_{L^{\infty}(\Omega)}^{p^{\prime}} C_{\Omega}^{p} \int_{\Omega}\left|\Phi_{1}\right|^{p} \omega_{1} d x+\left\|h_{2}\right\|_{L^{\infty}(\Omega)}^{p^{\prime}} \int_{\Omega}|\Phi|^{p} \omega_{1} d x\right] .
\end{aligned}
$$

By condition (H1), we have $F_{j} u_{m_{k}}(x)=\mathcal{A}_{j}\left(x, u_{m_{k}}(x), \nabla u_{m_{k}}(x)\right) \rightarrow \mathcal{A}_{j}(x, u(x), \nabla u(x))=F_{j} u(x)$, as $m_{k} \rightarrow+\infty$. Therefore, by the Lebesgue Dominated Convergence Theorem, we obtain $\left\|F_{j} u_{m_{k}}-F_{j} u\right\|_{L^{p^{\prime}}\left(\Omega, \omega_{1}\right)} \rightarrow 0$, that is, $F_{j} u_{m_{k}} \rightarrow F_{j} u$ in $L^{p^{\prime}}\left(\Omega, \omega_{1}\right)$. We conclude from the Convergence Principle in Banach spaces (see Proposition 10.13 in [20]) that

$$
F_{j} u_{m} \rightarrow F_{j} u \text { in } L^{p^{\prime}}\left(\Omega, \omega_{1}\right)
$$

Step 2. We define the operator $G_{j}: W_{0}^{1, p}\left(\Omega, \omega_{1}, \omega_{2}\right) \rightarrow L^{q^{\prime}}\left(\Omega, \nu_{1}\right)$ by $\left(G_{j} u\right)(x)=\mathcal{B}_{j}(x, u(x), \nabla u(x))$.

This operator is continuous and bounded. In fact,

(i) Using (H8), Remark 2.2(i) and Theorem 2.2 (since $\omega_{1} \in A_{p}$ ) we obtain

$$
\begin{aligned}
\left\|G_{j} u\right\|_{L^{q^{\prime}}\left(\Omega, \nu_{1}\right)}^{q^{\prime}} & =\int_{\Omega}\left|G_{j} u(x)\right|^{q^{\prime}} \nu_{1} d x=\int_{\Omega}\left|\mathcal{B}_{j}(x, u, \nabla u)\right|^{q^{\prime}} \nu_{1} d x \leq \int_{\Omega}\left(K_{2}+g_{1}|u|^{q / q^{\prime}}+g_{2}|\nabla u|^{q / q^{\prime}}\right)^{q^{\prime}} \nu_{1} d x \\
& \leq C_{q} \int_{\Omega}\left[\left(K_{2}^{q^{\prime}}+g_{1}^{q^{\prime}}|u|^{q}+g_{2}^{q^{\prime}}|\nabla u|^{q}\right) \nu_{1}\right] d x=C_{q}\left[\int_{\Omega} K_{2}^{q^{\prime}} \nu_{1} d x+\int_{\Omega} g_{1}^{q^{\prime}}|u|^{q} \nu_{1} d x+\int_{\Omega} g_{2}^{q^{\prime}}|\nabla u|^{q} \nu_{1} d x\right] \\
& \leq C_{q}\left(\left\|K_{2}\right\|_{L^{q^{\prime}\left(\Omega, \nu_{1}\right)}}^{q^{\prime}}+\left\|g_{1}\right\|_{L^{\infty}(\Omega)}^{q^{\prime}}\|u\|_{L^{q}\left(\Omega, \nu_{1}\right)}^{q}+\left\|g_{2}\right\|_{L^{\infty}(\Omega)}^{q^{\prime}}\||\nabla u|\|_{L^{q}\left(\Omega, \nu_{1}\right)}^{q}\right) \\
& \leq C_{q}\left(\left\|K_{2}\right\|_{L^{q^{\prime}\left(\Omega, \nu_{1}\right)}}^{q^{\prime}}+\left\|g_{1}\right\|_{L^{\infty}(\Omega)}^{q^{\prime}} C_{p, q}^{q}\|u\|_{L^{p}\left(\Omega, \omega_{1}\right)}^{q}+C_{p, q}^{q}\left\|g_{2}\right\|_{L^{\infty}(\Omega)}^{q^{\prime}}\||\nabla u|\|_{L^{p}\left(\Omega, \omega_{1}\right)}^{q}\right) \\
& \leq C_{q}\left(\left\|K_{2}\right\|_{L^{q^{\prime}\left(\Omega, \nu_{1}\right)}}^{q^{\prime}}+C_{p, q}^{q}\left(C_{\Omega}^{q}\left\|g_{1}\right\|_{L^{\infty}(\Omega)}^{q^{\prime}}+\left\|g_{2}\right\|_{L^{\infty}(\Omega)}^{q^{\prime}}\right)\|u\|_{W_{0}^{1, p}\left(\Omega, \omega_{1}, \omega_{2}\right)}^{q}\right)
\end{aligned}
$$

where $C_{q}$ depends only on $q$. Therefore, from (6) we obtain

$$
\left\|G_{j} u\right\|_{L^{q^{\prime}}\left(\Omega, \nu_{1}\right)} \leq C_{q}^{1 / q^{\prime}}\left(\left\|K_{2}\right\|_{L^{q^{\prime}}\left(\Omega, \nu_{1}\right)}+C_{p, q}^{q-1}\left(C_{\Omega}^{q-1}\left\|g_{1}\right\|_{L^{\infty}(\Omega)}+\left\|g_{2}\right\|_{L^{\infty}(\Omega)}\right)\|u\|_{W_{0}^{1, p}\left(\Omega, \omega_{1}, \omega_{2}\right)}^{q-1}\right) .
$$

(ii) Let $u_{m} \rightarrow u$ in $W_{0}^{1, p}\left(\Omega, \omega_{1}, \omega_{2}\right)$ as $m \rightarrow \infty$. We need to show that $G_{j} u_{m} \rightarrow G_{j} u$ in $L^{q^{\prime}}\left(\Omega, \nu_{1}\right)$. We will apply the Lebesgue Dominated Theorem. If $u_{m} \rightarrow u$ in $W_{0}^{1, p}\left(\Omega, \omega_{1}, \omega_{2}\right)$, then $\left|\nabla u_{m}\right| \rightarrow|\nabla u|$ in $L^{p}\left(\Omega, \omega_{1}\right)$. Using Theorem 2.1, there exist a subsequence $\left\{u_{m_{k}}\right\}$ and a function $\Phi_{1} \in L^{p}\left(\Omega, \omega_{1}\right)$ such that $D_{j} u_{m_{k}}(x) \rightarrow D_{j} u(x)$ a.e. in $\Omega$ and $\left|\nabla u_{m_{k}}(x)\right| \leq \Phi_{1}(x)$ a.e. in $\Omega$. Then, by Remark 2.2(i) and Theorem 2.2 (with $k=1$ ) we obtain

$$
\left\|u_{m_{k}}\right\|_{L^{q}\left(\Omega, \nu_{1}\right)} \leq C_{p, q}\left\|u_{m_{k}}\right\|_{L^{p}\left(\Omega, \omega_{1}\right)} \leq C_{p, q} C_{\Omega}\left\|\left|\nabla u_{m_{k}}\right|\right\|_{L^{p}\left(\Omega, \omega_{1}\right)} \leq C_{p, q} C_{\Omega}\left\|\Phi_{1}\right\|_{L^{p}\left(\Omega, \omega_{1}\right)} .
$$

Next, applying (H8) and Remark 2.2(i) we obtain

$$
\begin{aligned}
\left\|G_{j} u_{m_{k}}-G_{j} u\right\|_{L^{q^{\prime}}\left(\Omega, \nu_{1}\right)}^{q^{\prime}} & =\int_{\Omega}\left|G_{j} u_{m_{k}}(x)-G_{j} u(x)\right|^{q^{\prime}} \nu_{1} d x=\int_{\Omega}\left|\mathcal{B}_{j}\left(x, u_{m_{k}}, \nabla u_{m_{k}}\right)-\mathcal{B}_{j}(x, u, \nabla u)\right|^{q^{\prime}} \nu_{1} d x \\
& \leq C_{q} \int_{\Omega}\left(\left|\mathcal{B}_{j}\left(x, u_{m_{k}}, \nabla u_{m_{k}}\right)\right|^{q^{\prime}}+\left|\mathcal{B}_{j}(x, u, \nabla u)\right|^{q^{\prime}}\right) \nu_{1} d x \\
& \leq C_{q}\left[\int_{\Omega}\left(K_{2}+g_{1}\left|u_{m_{k}}\right|^{q / q^{\prime}}+g_{2}\left|\nabla u_{m_{k}}\right|^{q / q^{\prime}}\right)^{q^{\prime}} \nu_{1} d x+\int_{\Omega}\left(K_{2}+g_{1}|u|^{q / q^{\prime}}+g_{2}|\nabla u|^{q / q^{\prime}}\right)^{q^{\prime}} \nu_{1} d x\right]
\end{aligned}
$$




$$
\begin{aligned}
\leq & C_{q}\left[\int_{\Omega} K_{2}^{q^{\prime}} \nu_{1} d x+\left\|g_{1}\right\|_{L^{\infty}(\Omega)}^{q^{\prime}} \int_{\Omega}\left|u_{m_{k}}\right|^{q} \nu_{1} d x+\left\|g_{2}\right\|_{L^{\infty}(\Omega)}^{q^{\prime}} \int_{\Omega}\left|\nabla u_{m_{k}}\right|^{q} \nu_{1} d x\right. \\
& \left.+\int_{\Omega} K_{2}^{q^{\prime}} \nu_{1} d x+\left\|g_{1}\right\|_{L^{\infty}(\Omega)}^{q^{\prime}} \int_{\Omega}|u|^{q} \nu_{1} d x+\left\|g_{2}\right\|_{L^{\infty}(\Omega)}^{q^{\prime}} \int_{\Omega}|\nabla u|^{q} \nu_{1} d x\right] \\
\leq & 2 C_{q}\left[\left\|K_{2}\right\|_{L^{q^{\prime}}\left(\Omega, \nu_{1}\right)}+\left\|g_{1}\right\|_{L^{\infty}(\Omega)}^{q^{\prime}} C_{p, q}^{q} C_{\Omega}^{q}\|\Phi\|_{L^{p}\left(\Omega, \omega_{1}\right)}^{p / q}+\left\|g_{2}\right\|_{L^{\infty}(\Omega)}^{q^{\prime}}\|\Phi\|_{L^{p}\left(\Omega, \omega_{1}\right)}^{p / q}\right] .
\end{aligned}
$$

By condition (H5), we have $G_{j} u_{m_{k}}(x)=\mathcal{B}_{j}\left(x, u_{m_{k}}(x), \nabla u_{m_{k}}(x)\right) \rightarrow \mathcal{B}_{j}(x, u(x), \nabla u(x))=G_{j} u(x)$, as $m_{k} \rightarrow+\infty$. Hence, by the Lebesgue Dominated Convergence Theorem, we obtain $\left\|G_{j} u_{m_{k}}-G_{j} u\right\|_{L^{q^{\prime}}\left(\Omega, \nu_{1}\right)} \rightarrow 0$, i.e., $G_{j} u_{m_{k}} \rightarrow G_{j} u$ in $L^{q^{\prime}}\left(\Omega, \nu_{1}\right)$. We conclude from the Convergence Principle in Banach spaces (see Proposition 10.13 in [20]) that

$$
G_{j} u_{m} \rightarrow G_{j} u \text { in } L^{q^{\prime}}\left(\Omega, \nu_{1}\right)
$$

Step 3. We define the operator $H: W_{0}^{1, p}\left(\Omega, \omega_{1}, \omega_{2}\right) \rightarrow L^{s^{\prime}}\left(\Omega, \nu_{2}\right)$ by $(H u)(x)=\mathcal{H}(x, u(x), \nabla u(x))$. We also have that the operator $H$ is continuous and bounded. In fact,

(i) Using (H12), Remark 2.2(ii) and Theorem 2.2 we obtain

$$
\begin{aligned}
& \|H u\|_{L^{s^{\prime}}\left(\Omega, \nu_{2}\right)}^{s^{\prime}}=\int_{\Omega}|H u|^{s^{\prime}} \nu_{2} d x=\int_{\Omega}|\mathcal{H}(x, u, \nabla u)|^{s^{\prime}} \nu_{2} d x \leq \int_{\Omega}\left(K_{3}+h_{2}|u|^{s / s^{\prime}}+h_{3}|\nabla u|^{s / s^{\prime}}\right)^{s^{\prime}} \nu_{2} d x \\
& \leq C_{s} \int_{\Omega}\left(K_{3}^{s^{\prime}}+h_{2}^{s^{\prime}}|u|^{s}+h_{3}^{s^{\prime}}|\nabla u|^{s}\right) \nu_{2} d x \\
& \left.\leq C_{s} \int_{\Omega} K_{3}^{s^{\prime}} \nu_{2} d x+\left\|h_{2}\right\|_{L^{\infty}(\Omega)}^{s^{\prime}} \int_{\Omega}|u|^{s} \nu_{2} d x+\left\|h_{2}\right\|_{L^{\infty}(\Omega)}^{s^{\prime}} \int_{\Omega}|\nabla u|^{s} \nu_{2} d x\right] \\
& \leq C_{s}\left(\left\|K_{3}\right\|_{L^{s^{\prime}}\left(\Omega, \nu_{2}\right)}^{s^{\prime}}+\left\|h_{2}\right\|_{L^{\infty}(\Omega)}^{s^{\prime}} C_{p, s}^{s}\|u\|_{L^{p}\left(\Omega, \omega_{1}\right)}^{s}+\left\|h_{3}\right\|_{L^{\infty}(\Omega)}^{s^{\prime}} C_{p, s}^{s}\||\nabla u|\|_{L^{p}\left(\Omega, \omega_{1}\right)}^{s}\right) \\
& \leq C_{s}\left(\left\|K_{3}\right\|_{L^{s^{\prime}}\left(\Omega, \nu_{2}\right)}^{s^{\prime}}+\left\|h_{2}\right\|_{L^{\infty}(\Omega)}^{s^{\prime}} C_{p, s}^{s} C_{\Omega}^{s}\|\nabla u\|_{L^{p}\left(\Omega, \omega_{1}\right)}^{s}+\left\|h_{3}\right\|_{L^{\infty}(\Omega)}^{s^{\prime}} C_{p, s}^{s}\||\nabla u|\|_{L^{p}\left(\Omega, \omega_{1}\right)}^{s}\right) \\
& \leq C_{s}\left(\left\|K_{3}\right\|_{L^{s^{\prime}\left(\Omega, \nu_{2}\right)}}^{s^{\prime}}+C_{p, s}^{s}\left(C_{\Omega}^{s}\left\|h_{2}\right\|_{L^{\infty}(\Omega)}^{s^{\prime}}+\left\|h_{3}\right\|_{L^{\infty}(\Omega)}^{s^{\prime}}\right)\|u\|_{W_{0}^{1, p}\left(\Omega, \omega_{1}, \omega_{2}\right)}^{s}\right),
\end{aligned}
$$

where the constant $C_{s}$ depends only on $s$. Hence, from (8), we obtain

$$
\|H u\|_{L^{s^{\prime}}\left(\Omega, \nu_{2}\right)} \leq C_{s}\left[\left\|K_{3}\right\|_{L^{s^{\prime}}\left(\Omega, \nu_{2}\right)}+C_{p, s}^{s-1}\left(C_{\Omega}^{s-1}\left\|h_{2}\right\|_{L^{\infty}(\Omega)}+\left\|h_{3}\right\|_{L^{\infty}(\Omega)}\right)\|u\|_{W_{0}^{1, p}\left(\Omega, \omega_{1}, \omega_{2}\right)}^{s-1}\right] .
$$

(ii) Applying (H12) and Remark 2.2(ii), by the same argument used in Step 1(ii), we obtain analogously, if $u_{m} \rightarrow u$ in $W_{0}^{1, p}\left(\Omega, \omega_{1}, \omega_{2}\right)$ then

$$
H u_{m} \rightarrow H u, \text { in } L^{s^{\prime}}\left(\Omega, \nu_{2}\right) \text {. }
$$

Step 4. We define the operator $J: W_{0}^{1, p}\left(\Omega, \omega_{1}, \omega_{2}\right) \rightarrow L^{p^{\prime}}\left(\Omega, \omega_{2}\right)$ by $(J u)(x)=|u(x)|^{p-2} u(x)$. We also have that the operator $J$ is continuous and bounded. In fact:

(i) For all $u \in W_{0}^{1, p}\left(\Omega, \omega_{1}, \omega_{2}\right)$, we have

$$
\|J u\|_{L^{p^{\prime}\left(\Omega, \omega_{2}\right)}}^{p^{\prime}}=\int_{\Omega}|J u|^{p^{\prime}} \omega_{2} d x=\int_{\Omega}|u|^{(p-1) p^{\prime}} \omega_{2} d x=\int_{\Omega}|u|^{p} \omega_{2} d x \leq\|u\|_{W_{0}^{1, p}\left(\Omega, \omega_{1}, \omega_{2}\right)}^{p} .
$$

(ii) Let $u_{m} \rightarrow u$ in $W_{0}^{1, p}\left(\Omega, \omega_{1}, \omega_{2}\right)$. Then $u_{m} \rightarrow u$ in $L^{p}\left(\Omega, \omega_{2}\right)$. Using Theorem 2.1 , there exist a subsequence $\left\{u_{m}\right\}$ and a function $\Phi_{2} \in L^{p}\left(\Omega, \omega_{2}\right)$ such that $u_{m_{k}}(x) \rightarrow u(x)$ a.e. in $\Omega$ and $\left|u_{m_{k}}(x)\right| \leq \Phi_{2}(x)$ a.e. in $\Omega$. Next, applying Proposition 2.1(a), we have

$$
\begin{aligned}
\left\|J u_{m_{k}}-J u\right\|_{L^{p^{\prime}\left(\Omega, \omega_{2}\right)}}^{p^{\prime}} & =\int_{\Omega}\left|J u_{m_{k}}-J u\right|^{p^{\prime}} \omega_{2} d x=\left.\int_{\Omega}|| u_{m_{k}}\right|^{p-2} u_{m_{k}}-\left.|u|^{p-2} u\right|^{p^{\prime}} \omega_{2} d x \\
& \leq \int_{\Omega}\left[C_{p}\left|u_{m_{k}}-u\right|\left(\left|u_{m_{k}}\right|+|u|\right)^{p-2}\right]^{p^{\prime}} \omega_{2} d x \leq C_{p}^{p^{\prime}} \int_{\Omega}\left|u_{m_{k}}-u\right|^{p^{\prime}}\left(\left|u_{m_{k}}\right|+|u|\right)^{(p-2) p^{\prime}} \omega_{2} d x \\
& \leq 2^{(p-2) p^{\prime}} C_{p}^{p^{\prime}} \int_{\Omega}\left|u_{m_{k}}-u\right|^{p^{\prime}} \Phi_{2}^{(p-2) p^{\prime}} \omega_{2} d x \\
& \leq 2^{(p-2) p^{\prime}} C_{p}^{p^{\prime}}\left(\int_{\Omega}\left|u_{m_{k}}-u\right|^{p^{\prime}\left(p / p^{\prime}\right)} \omega_{2} d x\right)^{p^{\prime} / p}\left(\int_{\Omega} \Phi_{2}^{(p-2) p^{\prime} p /\left(p-p^{\prime}\right)} \omega_{2} d x\right)^{\left(p-p^{\prime}\right) / p} \\
& =2^{(p-2) p^{\prime}} C_{p}^{p^{\prime}}\left(\int_{\Omega}\left|u_{m_{k}}-u\right|^{p} \omega_{2} d x\right)^{p^{\prime} / p}\left(\int_{\Omega} \Phi_{2}^{p} \omega_{2} d x\right)^{\left(p-p^{\prime}\right) / p}
\end{aligned}
$$




$$
=2^{(p-2) p^{\prime}} C_{p}^{p^{\prime}}\left\|u_{m_{k}}-u\right\|_{L^{p}\left(\Omega, \omega_{2}\right)}^{p^{\prime}}\left\|\Phi_{2}\right\|_{L^{p}\left(\Omega, \omega_{2}\right)}^{p-p^{\prime}} .
$$

Hence $\left\|J u_{m_{k}}-J u\right\|_{L^{p^{\prime}\left(\Omega, \omega_{2}\right)}} \rightarrow 0$ as $m_{k} \rightarrow \infty$. We conclude from the Convergence Principle in Banach spaces that

$$
J u_{m} \rightarrow J u \text { in } L^{p^{\prime}}\left(\Omega, \omega_{2}\right) .
$$

Step 5. Since $\frac{f_{0}}{\nu_{1}} \in L^{q^{\prime}}\left(\Omega, \nu_{1}\right)$ and $\frac{f_{j}}{\omega_{1}} \in L^{p^{\prime}}\left(\Omega, \omega_{1}\right)(j=1, \ldots, n)$ then $\mathbf{T} \in\left[W_{0}^{1, p}\left(\Omega, \omega_{1}, \omega_{2}\right)\right]^{*}$. Moreover, by Remark 2.2(i), we have

$$
\begin{aligned}
|\mathbf{T}(\varphi)| & \leq \int_{\Omega}\left|f_{0} \| \varphi\right| d x+\sum_{j=1}^{n} \int_{\Omega}\left|f_{j}\right|\left|D_{j} \varphi\right| d x=\int_{\Omega} \frac{\left|f_{0}\right|}{\nu_{1}}|\varphi| \nu_{1} d x+\sum_{j=1}^{n} \int_{\Omega} \frac{\left|f_{j}\right|}{\omega_{1}}\left|D_{j} \varphi\right| \omega_{1} d x \\
& \leq\left\|f_{0} / \nu_{1}\right\|_{L^{q^{\prime}}\left(\Omega, \nu_{1}\right)}\|\varphi\|_{L^{q}\left(\Omega, \nu_{1}\right)}+\left(\sum_{j=1}^{n}\left\|f_{j} / \omega_{1}\right\|_{L^{p^{\prime}}\left(\Omega, \omega_{1}\right)}\right)\||\nabla \varphi|\|_{L^{p}\left(\Omega, \omega_{1}\right)} \\
& \leq\left(C_{p, q}\left\|f_{0} / \nu_{1}\right\|_{L^{q^{\prime}}\left(\Omega, \nu_{1}\right)}+\sum_{j=1}^{n}\left\|f_{j} / \omega_{1}\right\|_{L^{p^{\prime}}\left(\Omega, \omega_{1}\right)}\right)\|\varphi\|_{W_{0}^{1, p}\left(\Omega, \omega_{1}, \omega_{2}\right)}
\end{aligned}
$$

Also, we have

$$
\begin{aligned}
|\mathbf{B}(u, \varphi)| & \leq\left|\mathbf{B}_{\mathbf{1}}(u, \varphi)\right|+\left|\mathbf{B}_{\mathbf{2}}(u, \varphi)\right|+\left|\mathbf{B}_{3}(u, \varphi)\right|+\left|\mathbf{B}_{4}(u, \varphi)\right| \\
& \leq \int_{\Omega}|\mathcal{A}(x, u, \nabla u)||\nabla \varphi| \omega_{1} d x+\int_{\Omega}|\mathcal{B}(x, u, \nabla u)||\nabla \varphi| \nu_{1} d x+\int_{\Omega}|\mathcal{H}(x, u, \nabla u)| \nu_{2}+\int_{\Omega}|u|^{p-1}|\varphi| \omega_{2} d x .
\end{aligned}
$$

From (11), we get by using (H4), Theorem 2.2 (with $k=1$ ) and Remark 2.1,

$$
\begin{aligned}
\int_{\Omega}|\mathcal{A}(x, u, \nabla u)||\nabla \varphi| \omega_{1} d x & \leq \int_{\Omega}\left(K_{1}+h_{1}|u|^{p / p^{\prime}}+h_{2}|\nabla u|^{p / p^{\prime}}\right)|\nabla \varphi| \omega_{1} d x \\
& \leq\left\|K_{1}\right\|_{L^{p^{\prime}\left(\Omega, \omega_{1}\right)}}\||\nabla \varphi|\|_{L^{p}\left(\Omega, \omega_{1}\right)}+\left\|h_{1}\right\|_{L^{\infty}(\Omega)}\|u\|_{L^{p}\left(\Omega, \omega_{1}\right)}^{p / p^{\prime}}\||\nabla \varphi|\|_{L^{p}\left(\Omega, \omega_{1}\right)} \\
& +\left\|h_{2}\right\|_{L^{\infty}(\Omega)}\||\nabla u|\|_{L^{p}\left(\Omega, \omega_{1}\right)}^{p / p^{\prime}}\||\nabla \varphi|\|_{L^{p}\left(\Omega, \omega_{1}\right)} \\
& \leq\left(\left\|K_{1}\right\|_{L^{p^{\prime}\left(\Omega, \omega_{1}\right)}}+\left(C_{\Omega}^{1 / p^{\prime}}\left\|h_{1}\right\|_{L^{\infty}(\Omega)}+\left\|h_{2}\right\|_{L^{\infty}(\Omega)}\right)\|u\|_{W_{0}^{1, p}\left(\Omega, \omega_{1}, \omega_{2}\right)}^{p / p^{\prime}}\right)\|\varphi\|_{W_{0}^{1, p}\left(\Omega, \omega_{1}, \omega_{2}\right)},
\end{aligned}
$$

and by (H8) and Remark 2.2(i), we have

$$
\begin{aligned}
\int_{\Omega}|\mathcal{B}(x, u, \nabla u)||\nabla \varphi| \nu_{1} d x \leq & \int_{\Omega}\left(K_{2}+g_{1}|u|^{q / q^{\prime}}+g_{2}|\nabla u|^{q / q^{\prime}}\right)|\nabla \varphi| \nu_{1} d x \\
\leq & \left\|K_{2}\right\|_{L^{q^{\prime}}\left(\Omega, \nu_{1}\right)}\||\nabla \varphi|\|_{L^{q}\left(\Omega, \nu_{1}\right)}+\left\|g_{1}\right\|_{L^{\infty}(\Omega)}\|u\|_{L^{q}\left(\Omega, \nu_{1}\right)}^{q q^{\prime}}\||\nabla \varphi|\|_{L^{q}\left(\Omega, \nu_{1}\right)} \\
& +\left\|g_{2}\right\|_{L^{\infty}(\Omega)}\||\nabla u|\|_{L^{q}\left(\Omega, \nu_{1}\right)}^{q / q^{\prime}}\||\nabla \varphi|\|_{L^{q}\left(\Omega, \nu_{1}\right)} \\
\leq & C_{p, q}\left\|K_{2}\right\|_{L^{q^{\prime}\left(\Omega, \nu_{1}\right)}}\||\nabla \varphi|\|_{L^{p}\left(\Omega, \omega_{1}\right)}+C_{p, q}^{q-1}\left\|g_{1}\right\|_{L^{\infty}(\Omega)}\|u\|_{L^{p}\left(\Omega, \omega_{1}\right)}^{q-1} C_{p, q}\||\nabla \varphi|\|_{L^{p}\left(\Omega, \omega_{1}\right)} \\
& +\left\|g_{2}\right\|_{L^{\infty}(\Omega)} C_{p, q}^{q-1}\||\nabla u|\|_{L^{p}\left(\Omega, \omega_{1}\right)}^{q-1} C_{p, q}\||\nabla \varphi|\|_{L^{p}\left(\Omega, \omega_{1}\right)} \\
\leq & {\left[C_{p, q}\left\|K_{2}\right\|_{L^{q^{\prime}}\left(\Omega, \nu_{1}\right)}+\left(C_{p, q}^{q}\left\|g_{1}\right\|_{L^{\infty}(\Omega)}+C_{p, q}^{q}\left\|g_{2}\right\|_{L^{\infty}(\Omega)}\right)\|u\|_{W_{0}^{1, p}\left(\Omega, \omega_{1}, \omega_{2}\right)}^{q-1}\right]\|\varphi\|_{W_{0}^{1, p}\left(\Omega, \omega_{1}, \omega_{2}\right)}, }
\end{aligned}
$$

and by (H12) and Remark 2.2(ii), we obtain

$$
\begin{aligned}
& \int_{\Omega}|\mathcal{H}(x, u, \nabla u)||\varphi| \nu_{2} d x \leq \int_{\Omega}\left(K_{3}+h_{2}|u|^{s / s^{\prime}}+h_{3}|\nabla u|^{s / s^{\prime}}\right)|\varphi| \nu_{2} d x \\
& \leq \int_{\Omega} K_{3}|\varphi| \nu_{2} d x+\left\|h_{2}\right\|_{L^{\infty}(\Omega)} \int_{\Omega}|u|^{s / s^{\prime}}|\varphi| \nu_{2} d x+\left\|h_{3}\right\|_{L^{\infty}(\Omega)} \int_{\Omega}|\nabla u|^{s / s^{\prime}}|\varphi| \nu_{2} d x \\
& \leq\left\|K_{3}\right\|_{L^{s^{\prime}\left(\Omega, \nu_{2}\right)}}\|\varphi\|_{L^{s}\left(\Omega, \nu_{2}\right)}+\left\|h_{2}\right\|_{L^{\infty}(\Omega)}\|u\|_{L^{s}\left(\Omega, \nu_{2}\right)}^{s / s^{\prime}}\|\varphi\|_{L^{s}\left(\Omega, \nu_{2}\right)} \\
& \leq C_{p, s}\left\|K_{3}\right\|_{L^{s^{\prime}}(\Omega)}\|\varphi\|_{L^{p}\left(\Omega, \omega_{1}\right)}+\left\|h_{2}\right\|_{L^{\infty}(\Omega)} C_{p, s}^{s-1}\|u\|_{L^{p}\left(\Omega, \omega_{1}\right)}^{s-1} C_{p, s}\|\varphi\|_{L^{p}\left(\Omega, \omega_{1}\right)} \\
&+\left\|h_{3}\right\|_{L^{\infty}(\Omega)} C_{p, s}^{s-1}\||\nabla u|\|_{L^{p}\left(\Omega, \omega_{1}\right)}^{s-1} C_{p, s}\|\varphi\|_{L^{p}\left(\Omega, \omega_{1}\right)} \\
& \leq {\left[C_{p, s}\left\|K_{3}\right\|_{L^{s^{\prime}}\left(\Omega, \nu_{2}\right)}+C_{p, s}^{s}\left(\left\|h_{2}\right\|_{L^{\infty}(\Omega)}+\left\|h_{3}\right\|_{L^{\infty}(\Omega)}\right)\|u\|_{W_{0}^{1, p}\left(\Omega, \omega_{1}, \omega_{2}\right)}^{s-1}\right]\|\varphi\|_{W_{0}^{1, p}\left(\Omega, \omega_{1}, \omega_{2}\right)}, \text { and } } \\
& \int_{\Omega}|u|^{p-1}|\varphi| \omega_{2} d x \leq\left(\int_{\Omega}|u|^{p} \omega_{2} d x\right)^{1 / p^{\prime}}\left(\int_{\Omega}|\varphi|^{p} \omega_{2} d x\right)^{1 / p} \leq C_{\Omega}\|u\|_{W_{0}^{1, p}\left(\Omega, \omega_{1}, \omega_{2}\right)}^{p-1}\|\varphi\|_{W_{0}^{1, p}\left(\Omega, \omega_{1}, \omega_{2}\right)} .
\end{aligned}
$$


Hence, by (11), for all $u, \varphi \in W_{0}^{1, p}\left(\Omega, \omega_{1}, \omega_{2}\right)$ it holds that

$$
\begin{aligned}
|\mathbf{B}(u, \varphi)| \leq & {\left[\left\|K_{1}\right\|_{L^{p^{\prime}}\left(\Omega, \omega_{1}\right)}+\left\|h_{1}\right\|_{L^{\infty}(\Omega)}\|u\|_{W_{0}^{1, p}\left(\Omega, \omega_{1}, \omega_{2}\right)}^{p-1}+C_{p, q}\left\|K_{2}\right\|_{L^{q^{\prime}}\left(\Omega, \nu_{1}\right)}\right.} \\
& +C_{p, q}^{q}\left(\left\|g_{1}\right\|_{L^{\infty}(\Omega)}+\left\|g_{2}\right\|_{L^{\infty}(\Omega)}\right)\|u\|_{W_{0}^{1, p}\left(\Omega, \omega_{1}, \omega_{2}\right)}^{q-1}+C_{p, s}\left\|K_{3}\right\|_{L^{s^{\prime}}\left(\Omega, \nu_{2}\right)} \\
& \left.+C_{p, s}^{s}\left(\left\|h_{2}\right\|_{L^{\infty}(\Omega)}+\left\|h_{3}\right\|_{L^{\infty}(\Omega)}\right)\|u\|_{W_{0}^{1, p}\left(\Omega, \omega_{1}, \omega_{2}\right)}^{s-1}+C_{\Omega}\|u\|_{W_{0}^{1, p}\left(\Omega, \omega_{1}, \omega_{2}\right)}^{p-1}\right]\|\varphi\|_{W_{0}^{1, p}\left(\Omega, \omega_{1}, \omega_{2}\right)} .
\end{aligned}
$$

Since $\mathbf{B}(u,$.$) is linear, for each u \in W_{0}^{1, p}\left(\Omega, \omega_{1}, \omega_{2}\right)$, there exists a linear and continuous functional on $W_{0}^{1, p}\left(\Omega, \omega_{1}, \omega_{2}\right)$ denoted by $A u$ such that $(A u \mid \varphi)=\mathbf{B}(u, \varphi)$ for all $u, \varphi \in W_{0}^{1, p}\left(\Omega, \omega_{1}, \omega_{2}\right)$ (here $(f \mid x)$ denotes the value of the linear functional $f$ at the point $x$ ). Moreover, we have

$$
\begin{aligned}
\|A u\|_{*} \leq & \left\|K_{1}\right\|_{L^{p^{\prime}\left(\Omega, \omega_{1}\right)}}+\left\|h_{1}\right\|_{L^{\infty}(\Omega)}\|u\|_{W_{0}^{1, p}\left(\Omega, \omega_{1}, \omega_{2}\right)}^{p-1}+C_{p, q}\left\|K_{2}\right\|_{L^{q^{\prime}\left(\Omega, \nu_{1}\right)}}+C_{p, q}^{q}\left(\left\|g_{1}\right\|_{L^{\infty}(\Omega)}+\left\|g_{2}\right\|_{L^{\infty}(\Omega)}\right)\|u\|_{W_{0}^{1, p}\left(\Omega, \omega_{1}, \omega_{2}\right)}^{q-1} \\
& +C_{p, s}\left\|K_{3}\right\|_{L^{s^{\prime}}\left(\Omega, \nu_{2}\right)}+C_{p, s}^{s}\left(\left\|h_{2}\right\|_{L^{\infty}(\Omega)}+\left\|h_{3}\right\|_{L^{\infty}(\Omega)}\right)\|u\|_{W_{0}^{1, p}\left(\Omega, \omega_{1}, \omega_{2}\right)}^{s-1}+C_{\Omega}\|u\|_{W_{0}^{1, p}\left(\Omega, \omega_{1}, \omega_{2}\right)}^{p-1} .
\end{aligned}
$$

where $\|A u\|_{*}=\sup \left\{|(A u \mid \varphi)|=|B(u, \varphi)|: \varphi \in W_{0}^{1, p}\left(\Omega, \omega_{1}, \omega_{2}\right),\|\varphi\|_{W_{0}^{1, p}\left(\Omega, \omega_{1}, \omega_{2}\right)}=1\right\}$ is the norm of the operator $A u$. Hence, we obtain the operator

$$
\begin{aligned}
A: W_{0}^{1, p}\left(\Omega, \omega_{1}, \omega_{2}\right) \rightarrow\left[W_{0}^{1, p}\left(\Omega, \omega_{1}, \omega_{2}\right)\right]^{*} \\
u \mapsto A u .
\end{aligned}
$$

Consequently, problem (P) is equivalent to the operator equation $A u=\mathbf{T}, u \in W_{0}^{1, p}\left(\Omega, \omega_{1}, \omega_{2}\right)$.

Step 6. Using (H2), (H6), (H10) and Proposition 2.1(b), we obtain (for $\left.u_{1}, u_{2} \in W_{0}^{1, p}\left(\Omega, \omega_{1}, \omega_{2}\right), u_{1} \neq u_{2}\right)$

$$
\begin{aligned}
\left(A u_{1}-A u_{2} \mid u_{1}-u_{2}\right)= & \mathbf{B}\left(u_{1}, u_{1}-u_{2}\right)-\mathbf{B}\left(u_{2}, u_{1}-u_{2}\right) \\
= & \int_{\Omega}\left\langle\mathcal{A}\left(x, u_{1} \nabla u_{1}\right), \nabla\left(u_{1}-u_{2}\right)\right\rangle \omega_{1} d x+\int_{\Omega}\left\langle\mathcal{B}\left(x, u_{1}, \nabla u_{1}\right), \nabla\left(u_{1}-u_{2}\right)\right\rangle \nu_{1} d x \\
& +\int_{\Omega} \mathcal{H}\left(x, u_{1}, \nabla u_{1}\right)\left(u_{1}-u_{2}\right) \nu_{2} d x+\int_{\Omega}\left|u_{1}\right|^{p-2} u_{1}\left(u_{1}-u_{2}\right) \omega_{2} d x \\
& -\int_{\Omega}\left\langle\mathcal{A}\left(x, u_{2}, \nabla u_{2}\right), \nabla\left(u_{1}-u_{2}\right)\right\rangle \omega_{1} d x-\int_{\Omega}\left\langle\mathcal{B}\left(x, u_{2}, \nabla u_{2}, \nabla\left(u_{1}-u_{2}\right)\right\rangle \nu_{1} d x\right. \\
& -\int_{\Omega} \mathcal{H}\left(x, u_{2}, \nabla u_{2}\right)\left(u_{1}-u_{2}\right) \nu_{2} d x-\int_{\Omega}\left|u_{2}\right|^{p-2} u_{2}\left(u_{1}-u_{2}\right) \omega_{2} d x \\
= & \int_{\Omega}\left\langle\mathcal{A}\left(x, u_{1} \nabla u_{1}\right)-\mathcal{A}\left(x, u_{2}, \nabla u_{2}\right), \nabla\left(u_{1}-u_{2}\right)\right\rangle \omega_{1} d x \\
& +\int_{\Omega}\left\langle\mathcal{B}\left(x, u_{1}, \nabla u_{1}\right)-\mathcal{B}\left(x, u_{2}, \nabla u_{2}\right), \nabla\left(u_{1}-u_{2}\right)\right\rangle \nu_{1} d x \\
& +\int_{\Omega}\left(\mathcal{H}\left(x, u_{1}, \nabla u_{1}\right)-\mathcal{H}\left(x, u_{2}, \nabla u_{2}\right)\right)\left(u_{1}-u_{2}\right) \nu_{2} d x+\int_{\Omega}\left(\left|u_{1}\right|^{p-2} u_{1}-\left|u_{2}\right|^{p-2} u_{2}\right)\left(u_{1}-u_{2}\right) \omega_{2} d x \\
\geq & \theta_{1} \int_{\Omega}\left|\nabla\left(u_{1}-u_{2}\right)\right|^{p} \omega_{1} d x+\beta_{p} \int_{\Omega}\left(\left|u_{1}\right|+\left|u_{2}\right|\right)^{p-2}\left|u_{1}-u_{2}\right|^{2} \omega_{2} d x \\
\geq & \theta_{1} \int_{\Omega}\left|\nabla\left(u_{1}-u_{2}\right)\right|^{p} \omega_{1} d x+\beta_{p} \int_{\Omega}\left|u_{1}-u_{2}\right|^{p-2}\left|u_{1}-u_{2}\right|^{2} \omega_{2} d x \\
\geq & \theta_{1} \int_{\Omega}\left|\nabla\left(u_{1}-u_{2}\right)\right|^{p} \omega_{1} d x+\beta_{p} \int_{\Omega}\left|u_{1}-u_{2}\right|^{p} \omega_{2} d x \geq \gamma_{1} \| u_{1}-\left.u_{2}\right|_{W_{0}^{1, p}\left(\Omega, \omega_{1}, \omega_{2}\right)} ^{p} \\
&
\end{aligned}
$$

where $\gamma_{1}=\min \left\{\theta_{1}, \beta_{p}\right\}$. Therefore, the operator $A$ is strictly monotone. Moreover, from (H3),(H7), (H11), we obtain

$$
\begin{aligned}
(A u \mid u) & =\mathbf{B}(u, u)=\mathbf{B}_{\mathbf{1}}(u, u)+\mathbf{B}_{\mathbf{2}}(u, u)+\mathbf{B}_{3}(u, u)+\mathbf{B}_{4}(u, u) \\
& =\int_{\Omega}\langle\mathcal{A}(x, u, \nabla u), \nabla u\rangle \omega_{1} d x+\int_{\Omega}\langle\mathcal{B}(x, u, \nabla u), \nabla u\rangle \nu_{1} d x+\int_{\Omega} \mathcal{H}(x, u, \nabla u) u \nu_{2} d x+\int_{\Omega}|u|^{p} \omega_{2} d x \\
& \geq \lambda_{1} \int_{\Omega}|\nabla u|^{p} \omega_{1} d x+\lambda_{2} \int_{\Omega}|\nabla u|^{q} \nu_{1} d x+\Lambda_{2} \int_{\Omega}|u|^{q} \nu_{1} d x+\lambda_{3} \int_{\Omega}|\nabla u|^{s} \nu_{2} d x+\Lambda_{3} \int_{\Omega}|u|^{s} \nu_{2} d x+\int_{\Omega}|u|^{p} \omega_{2} d x \\
& \geq \lambda_{1} \int_{\Omega}|\nabla u|^{p} \omega_{1} d x+\int_{\Omega}|u|^{p} \omega_{2} d x \geq \gamma_{2}\|u\|_{W_{0}^{1, p}\left(\Omega, \omega_{1}\right), \omega_{2}}^{p},
\end{aligned}
$$

where $\gamma_{2}=\min \left\{\lambda_{1}, 1\right\}$. Hence, since $1<q, s<p<\infty$, we have

$$
\frac{(A u \mid u)}{\|u\|_{W_{0}^{1, p}\left(\Omega, \omega_{1}, \omega_{2}\right)}} \rightarrow+\infty, \text { as }\|u\|_{W_{0}^{1, p}\left(\Omega, \omega_{1}, \omega_{2}\right)} \rightarrow+\infty
$$


that is, $A$ is coercive.

Step 7. We need to show that the operator $A$ is continuous. Let $u_{m} \rightarrow u$ in $X$ as $m \rightarrow \infty$. We have,

$$
\begin{aligned}
\left|\mathbf{B}_{\mathbf{1}}\left(u_{m}, \varphi\right)-\mathbf{B}_{\mathbf{1}}(u, \varphi)\right| & \leq \sum_{j=1}^{n} \int_{\Omega}\left|\mathcal{A}_{j}\left(x, u_{m}, \nabla u_{m}\right)-\mathcal{A}_{j}(x, u, \nabla u)\left\|D_{j} \varphi\left|\omega_{1} d x=\sum_{j=1}^{n} \int_{\Omega}\right| F_{j} u_{m}-F_{j} u\right\| D_{j} \varphi\right| \omega_{1} d x \\
& \leq\left(\sum_{j=1}^{n}\left\|F_{j} u_{m}-F_{j} u\right\|_{L^{p^{\prime}}\left(\Omega, \omega_{1}\right)}\right)\||\nabla \varphi|\|_{L^{p}\left(\Omega, \omega_{1}\right)} \leq\left(\sum_{j=1}^{n}\left\|F_{j} u_{m}-F_{j} u\right\|_{L^{p^{\prime}}\left(\Omega, \omega_{1}\right)}\right)\|\varphi\|_{W_{0}^{1, p}\left(\Omega, \omega_{1}, \omega_{2}\right)},
\end{aligned}
$$

and by Remark 2.2(i), we get

$$
\begin{aligned}
\left|\mathbf{B}_{\mathbf{2}}\left(u_{m}, \varphi\right)-\mathbf{B}_{\mathbf{2}}(u, \varphi)\right| & \leq \sum_{j=1}^{n} \int_{\Omega}\left|\mathcal{B}_{j}\left(x, u_{m}, \nabla u_{m}\right)-\mathcal{B}_{j}(x, u, \nabla u)\left\|D_{j} \varphi\left|\nu_{1} d x=\sum_{j=1}^{n} \int_{\Omega}\right| G_{j} u_{m}-G_{j} u\right\| D_{j} \varphi\right| \nu_{1} d x \\
& \leq\left(\sum_{j=1}^{n}\left\|G_{j} u_{m}-G_{j} u\right\|_{L^{q^{\prime}}\left(\Omega, \nu_{1}\right)}\right)\||\nabla \varphi|\|_{L^{q}\left(\Omega, \nu_{1}\right)} \\
& \leq C_{p, q}\left(\sum_{j=1}^{n}\left\|G_{j} u_{m}-G_{j} u\right\|_{L^{q^{\prime}}\left(\Omega, \nu_{1}\right)}\right)\||\nabla \varphi|\|_{L^{p}\left(\Omega, \omega_{1}\right)} \\
& \leq C_{p, q}\left(\sum_{j=1}^{n}\left\|G_{j} u_{m}-G_{j} u\right\|_{L^{q^{\prime}}\left(\Omega, \nu_{1}\right)}\right)\|\varphi\|_{W_{0}^{1, p}\left(\Omega, \omega_{1}, \omega_{2}\right)}
\end{aligned}
$$

and by Remark 2.2(ii), we have

$$
\begin{aligned}
\left|\mathbf{B}_{3}\left(u_{m}, \varphi\right)-\mathbf{B}_{3}(u, \varphi)\right| & \leq \int_{\Omega}\left|\mathcal{H}\left(x, u_{m}, \nabla u_{m}\right)-\mathcal{H}(x, u, \nabla u)\right||\varphi| \nu_{2} d x=\int_{\Omega}\left|H u_{m}-H u\right||\varphi| \nu_{2} d x \\
& \leq\left\|H u_{m}-H u\right\|_{L^{s^{\prime}}\left(\Omega, \nu_{2}\right)}\|\varphi\|_{L^{s}\left(\Omega, \nu_{2}\right)} \leq C_{p, s}\left\|H u_{m}-H u\right\|_{L^{s^{\prime}}\left(\Omega, \nu_{2}\right)}\|\varphi\|_{L^{p}\left(\Omega, \omega_{1}\right)} \\
& \leq C_{p, s}\left\|H u_{m}-H u\right\|_{L^{s^{\prime}}\left(\Omega, \nu_{2}\right)}\|\varphi\|_{W_{0}^{1, p}\left(\Omega, \omega_{1}, \omega_{2}\right)},
\end{aligned}
$$

and by Step 4, we get

$$
\begin{aligned}
\left|\mathbf{B}_{4}\left(u_{m}, \varphi\right)-\mathbf{B}_{4}(u, \varphi)\right| & \leq\left.\int_{\Omega}|| u_{m}\right|^{p-2} u_{m}-|u|^{p-2} u|| \varphi\left|\omega_{2} d x=\int_{\Omega}\right| J u_{m}-J u|| \varphi \mid \omega_{2} d x \\
& \leq\left\|J u_{m}-J u\right\|_{L^{p^{\prime}}\left(\Omega, \omega_{2}\right)}\|\varphi\|_{W_{0}^{1, p}\left(\Omega, \omega_{1}, \omega_{2}\right)},
\end{aligned}
$$

for all $\varphi \in W_{0}^{1, p}\left(\Omega, \omega_{1}, \omega_{2}\right)$. Hence,

$$
\begin{aligned}
\left|\mathbf{B}\left(u_{m}, \varphi\right)-\mathbf{B}(u, \varphi)\right| \leq & \left|\mathbf{B}_{\mathbf{1}}\left(u_{m}, \varphi\right)-\mathbf{B}_{\mathbf{1}}(u, \varphi)\right|+\left|\mathbf{B}_{\mathbf{2}}\left(u_{m}, \varphi\right)-\mathbf{B}_{\mathbf{2}}(u, \varphi)\right| \\
& +\left|\mathbf{B}_{\mathbf{3}}\left(u_{m}, \varphi\right)-\mathbf{B}_{\mathbf{3}}(u, \varphi)\right|+\left|\mathbf{B}_{\mathbf{4}}\left(u_{m}, \varphi\right)-\mathbf{B}_{\mathbf{4}}(u, \varphi)\right| \\
\leq & {\left[\sum_{j=1}^{n}\left(\left\|F_{j} u_{m}-F_{j} u\right\|_{L^{p^{\prime}}\left(\Omega, \omega_{1}\right)}+C_{p, q}\left\|G_{j} u_{m}-G_{j} u\right\|_{L^{q^{\prime}}\left(\Omega, \nu_{1}\right)}\right)\right.} \\
& \left.+C_{p, s}\left\|H u_{m}-H u\right\|_{L^{s^{\prime}}\left(\Omega, \nu_{2}\right)}+\left\|J u_{m}-J u\right\|_{L^{p^{\prime}}\left(\Omega, \omega_{2}\right)}\right]\|\varphi\|_{W_{0}^{1, p}\left(\Omega, \omega_{1}, \omega_{2}\right)} .
\end{aligned}
$$

Then, we obtain

$$
\begin{aligned}
\left\|A u_{m}-A u\right\|_{*} \leq & \sum_{j=1}^{n}\left(\left\|F_{j} u_{m}-F_{j} u\right\|_{L^{p^{\prime}}\left(\Omega, \omega_{1}\right)}+C_{p, q}\left\|G_{j} u_{m}-G_{j} u\right\|_{L^{q^{\prime}}\left(\Omega, \nu_{1}\right)}\right) \\
& +C_{p, s}\left\|H u_{m}-H u\right\|_{L^{s^{\prime}}\left(\Omega, \nu_{2}\right)}+\left\|J u_{m}-J u\right\|_{L^{p^{\prime}}\left(\Omega, \omega_{2}\right)} .
\end{aligned}
$$

Hence, using (5), (7), (9) and (10) we have $\left\|A u_{m}-A u\right\|_{*} \rightarrow 0$ as $m \rightarrow+\infty$, that is, $A$ is continuous and this implies that $A$ is hemicontinuous. Therefore, by Theorem 3.1, the operator equation $A u=\mathbf{T}$ has a unique solution $u \in W_{0}^{1, p}\left(\Omega, \omega_{1}, \omega_{2}\right)$ and it is the unique solution for problem (P).

Step 8. Estimates for $\|u\|_{W_{0}^{1, p}\left(\Omega, \omega_{1}, \omega_{2}\right)}$. In particular, by setting $\varphi=u$ in Definition 2.3, we have

$$
\mathbf{B}(u, u)=\mathbf{B}_{1}(u, u)+\mathbf{B}_{2}(u, u)+\mathbf{B}_{3}(u, u)+\mathbf{B}_{4}(u, u)=\mathbf{T}(u) .
$$

Hence, using (H3),(H7), (H11) and Remark 2.2(ii) we obtain

$$
\mathbf{B}_{1}(u, u)+\mathbf{B}_{\mathbf{2}}(u, u)+\mathbf{B}_{3}(u, u)+\mathbf{B}_{\mathbf{4}}(u, u)=\int_{\Omega}\langle\mathcal{A}(x, u, \nabla u), \nabla u\rangle \omega_{1} d x+\int_{\Omega}\langle\mathbf{B}(x, u, \nabla u), \nabla u\rangle \nu_{1} d x
$$




$$
\begin{aligned}
& +\int_{\Omega} H(x, u, \nabla u) u \nu_{2} d x+\int_{\Omega}|u|^{p-2} u^{2} \omega_{2} \\
\geq & \lambda_{1} \int_{\Omega}|\nabla u|^{p} \omega_{1} d x+\lambda_{2} \int_{\Omega}|\nabla u|^{q} \nu_{1} d x+\Lambda_{2} \int_{\Omega}|u|^{q} \nu_{1} d x \\
& +\lambda_{3} \int_{\Omega}|\nabla u|^{s} \nu_{2} d x+\Lambda_{3} \int_{\Omega}|u|^{s} \nu_{2} d x+\int_{\Omega}|u|^{p} \omega_{2} d x \\
\geq & \lambda_{1} \int_{\Omega}|\nabla u|^{p} \omega_{1} d x+\int_{\Omega}|u|^{p} \omega_{2} d x \geq \gamma_{2}\|u\|_{W_{0}^{1, p}\left(\Omega, \omega_{1}, \omega_{2}\right)}^{p},
\end{aligned}
$$

and

$$
\begin{aligned}
\mathbf{T}(u) & =\int_{\Omega} f_{0} u d x+\sum_{j=1}^{n} \int_{\Omega} f_{j} D_{j} u d x \leq\left\|f_{0} / \nu_{1}\right\|_{L^{q^{\prime}}\left(\Omega, \nu_{1}\right)}\|u\|_{L^{q}\left(\Omega, \nu_{1}\right)}+\left(\sum_{j=1}^{n}\left\|f_{j} / \omega_{1}\right\|_{L^{p^{\prime}\left(\Omega, \omega_{1}\right)}}\right)\|\mid \nabla u\| \|_{L^{p}\left(\Omega, \omega_{1}\right)} \\
& \leq\left(C_{p, q}\left\|f_{0} / \nu_{1}\right\|_{L^{q^{\prime}}\left(\Omega, \nu_{1}\right)}+\sum_{j=1}^{n}\left\|f_{j} / \omega_{1}\right\|_{L^{p^{\prime}\left(\Omega, \omega_{1}\right)}}\right)\|u\|_{W_{0}^{1, p}\left(\Omega, \omega_{1}, \omega_{2}\right)}=M\|u\|_{W_{0}^{1, p}\left(\Omega, \omega_{1}, \omega_{2}\right)},
\end{aligned}
$$

where $M=C_{p, q}\left\|f_{0} / \nu_{1}\right\|_{L^{q^{\prime}}\left(\Omega, \nu_{1}\right)}+\sum_{j=1}^{n}\left\|f_{j} / \omega_{1}\right\|_{L^{p^{\prime}}\left(\Omega, \omega_{1}\right)}$. Hence, using (13) and (14) in (12), we obtain

$$
\gamma_{2}\|u\|_{W_{0}^{1, p}\left(\Omega, \omega_{1}, \omega_{2}\right)}^{p} \leq M\|u\|_{W_{0}^{1, p}\left(\Omega, \omega_{1}, \omega_{2}\right)} .
$$

Therefore,

$$
\|u\|_{W_{0}^{1, p}\left(\Omega, \omega_{1}, \omega_{2}\right)} \leq\left(\frac{M}{\gamma_{2}}\right)^{1 /(p-1)}=C\left(C_{p, q}\left\|f_{0} / \nu_{1}\right\|_{L^{q^{\prime}}\left(\Omega, \nu_{1}\right)}+\sum_{j=1}^{n}\left\|f_{j} / \omega_{1}\right\|_{L^{p^{\prime}}\left(\Omega, \omega_{1}\right)}\right)^{1 /(p-1)}
$$

where $C=\left(1 / \gamma_{2}\right)^{1 /(p-1)}$.

Example 3.1. Take $\Omega=\left\{(x, y) \in \mathbb{R}^{2}: x^{2}+y^{2}<1\right\}$ and consider the weight functions $\omega_{1}(x, y)=\left(x^{2}+y^{2}\right)^{-1 / 2}, \omega_{2}(x, y)=$ $\left(x^{2}+y^{2}\right)^{-3 / 2}, \nu_{1}(x, y)=\left(x^{2}+y^{2}\right)^{-1 / 3}$ and $\nu_{2}(x, y)=\left(x^{2}+y^{2}\right)^{-1}\left(\omega_{1}, \omega_{2} \in A_{4}, p=4, q=3\right.$ and $\left.s=2\right)$ and the function $\mathcal{A}: \Omega \times \mathbb{R} \times \mathbb{R}^{2} \rightarrow \mathbb{R}^{2}$ defined by $\mathcal{A}((x, y), \eta, \xi)=h_{1}(x, y)|\xi|^{2} \xi$, where $h_{1}(x, y)=2 \mathrm{e}^{\left(x^{2}+y^{2}\right)} ;$ and $\mathcal{B}: \Omega \times \mathbb{R} \times \mathbb{R}^{2} \rightarrow \mathbb{R}^{2}$ defined by $\mathcal{B}((x, y), \eta, \xi)=g_{2}(x, y)|\xi| \xi$, where $g_{2}(x, y)=2+\cos \left(x^{2}+y^{2}\right) ;$ and $\mathcal{H}: \Omega \times \mathbb{R} \times \mathbb{R}^{2} \rightarrow \mathbb{R}$ defined by $\mathcal{H}((x, y), \eta, \xi)=\eta h_{2}(x, y)$, where $h_{2}(x, y)=1+\cos ^{2}(x y)$. Let us consider the partial differential operator

$$
L u(x, y)=-\operatorname{div}\left(\mathcal{A}((x, y), \nabla u) \omega_{1}(x, y)+\mathcal{B}((x, y), u, \nabla u) \nu_{1}(x, y)\right)+\mathcal{H}((x, y), u, \nabla u) \nu_{2}(x, y)+|u|^{2} u \omega_{2}(x, y) .
$$

Therefore, by Theorem 1.1, the problem

$$
(P)\left\{\begin{array}{l}
L u(x)=\frac{\cos (x y)}{\left(x^{2}+y^{2}\right)}-\frac{\partial}{\partial x}\left(\frac{\sin (x y)}{\left(x^{2}+y^{2}\right)}\right)-\frac{\partial}{\partial y}\left(\frac{\sin (x y)}{\left(x^{2}+y^{2}\right)}\right) \text { in } \Omega \\
u(x)=0 \text { on } \partial \Omega
\end{array}\right.
$$

has a unique solution $u \in W_{0}^{1,4}\left(\Omega, \omega_{1}, \omega_{2}\right)$.

\section{References}

[1] D. Bresch, J. Lemoine, F. Guíllen-Gonzalez, A note on a degenerate elliptic equation with applications for lakes and seas, Electron. J. Differential Equations 2004 (2004) Art\# 42.

[2] A. C. Cavalheiro, Existence and uniqueness of solutions for some degenerate nonlinear Dirichlet problems, J. Appl. Anal. 19 (2013) 41-54.

[3] A. C. Cavalheiro, Existence results for Dirichlet problems with degenerate p-Laplacian, Opuscula Math. 33 (2013) $439-453$.

[4] A. C. Cavalheiro, Topics on Degenerate Elliptic Equations, Lambert, Riga, 2018.

[5] M. Chipot, Elliptic Equations: An Introductory Course, Birkhäuser, Berlin, 2009.

[6] M. Colombo, Flows of Non-Smooth Vector Fields and Degenerate Elliptic Equations: With Applications to the Vlasov-Poisson and Semigeostrophic Systems, Edizioni della Normale, Pisa, 2017.

[7] P. Drábek, A. Kufner, F. Nicolosi, Quasilinear Elliptic Equations with Degenerations and Singularities, Walter de Gruyter, Berlin, 1997.

[8] E. Fabes, C. Kenig, R. Serapioni, The local regularity of solutions of degenerate elliptic equations, Comm. Partial Differential Equations 7 (1982) 77-116.

[9] S. Fučik, O. John, A. Kufner, Function Spaces, Noordhoff International Publ., Leyden, 1977.

[10] J. García-Cuerva, J. L. Rubio de Francia, Weighted Norm Inequalities and Related Topics, North-Holland, Amsterdam, 1985.

[11] D. Gilbarg, N. S. Trudinger, Elliptic Partial Equations of Second Order, 2nd Ed., Springer, New York, 1983.

[12] J. Heinonen, T. Kilpeläinen, O. Martio, Nonlinear Potential Theory of Degenerate Elliptic Equations, Clarendon Press, Oxford, 1993.

[13] A. Kufner, Weighted Sobolev Spaces, John Wiley \& Sons, New York, 1985.

[14] A. Kufner, B. Opic, How to define reasonably weighted Sobolev spaces, Comment. Math. Univ. Carolin. 25 (1984) $537-554$.

[15] B. Muckenhoupt, Weighted norm inequalities for the Hardy maximal function, Trans. Amer. Math. Soc. 165 (1972) $207-226$.

[16] B. Opic, A. Kufner, Hardy-Type Inequalities, Longman Scientific \& Technical, Harlow, 1990.

[17] M. Talbi, N. Tsouli, On the spectrum of the weighted p-Biharmonic operator with weight, Mediterr. J. Math. 4 (2007) 73-86.

[18] A. Torchinsky, Real-Variable Methods in Harmonic Analysis, Academic Press, San Diego, 1986.

[19] B.O. Turesson, Nonlinear Potential Theory and Weighted Sobolev Spaces, Springer, Berlin, 2000.

[20] E. Zeidler, Nonlinear Functional Analysis and its Applications, Vol. I, Springer, Berlin, 1990.

[21] E. Zeidler, Nonlinear Functional Analysis and its Applications, Vol. II/B, Springer, Berlin, 1990. 